\title{
Advanced Building Engineering: Deploying Mechatronics and Robotics in Architecture
}

\author{
Thomas Linner *, Christos Georgoulas, and Thomas Bock \\ Department of Architecture, Building Realization and Robotics Lab, Technical University \\ Munich, Germany \\ *Corresponding author (thomas.linner@br2.ar.tum.de)
}

\begin{abstract}
Purpose As robots are not yet widely accepted in Europe as service providers in home environments, the authors intent to establish the possibility to distribute robotic elements and mechatronic devices in order to fuse them with various subsystems of buildings. Thus, robotic service systems could support Activities of Daily Living (ADLs), becoming an invisible part of the building. The proposed approach combines and applies strategies of performance oriented architecture ${ }^{1}$, ubiquitous computing ${ }^{2}$ and service robotics ${ }^{3}$. Method Buildings and environments consist of a multitude of subsystems as for example walls, ceilings, furniture, service cores and many others. In order to realize the proposed vision of seamless robotic assistance, the authors, since 2008, extensively studied various subsystems, exploring how these could be fused with mechatronic and robotic elements. Currently, the research team enters Step 2 (Table 1). In the proposed paper, building subsystems that had been experimentally fused with mechatronic and robotic elements during Step 1 are described and evaluated. For each category, the authors present their implemented system, embedded into a 1:1 scale prototype experimental flat, which was developed for the realization of the proposed robotic environment. Additionally, the paper outlines: a) how the systems can support ADLs, b) architectural aspects, c) deployed sensor-actuator systems and d) the use of other basic technologies (Vision systems, Robot Operating System: ROS, etc.) during implementation. Results \& Discussion The evaluations showed that the fusion of building subsystems with robotic technology, in each category (Wall, Ceiling, Service Core, Cabinet, Seat, In-House Mobility - Table 1), holds the potential to create assistive systems that are acceptable in the home environment, and moreover able to assist a multitude of ADLs. The evaluations also revealed that modularity and safety issues should be addressed intensively in future research. The author's ultimate goal is to explore more building subsystems and finally integrate all systems explored during step 1 and step 2 to an integrated robotic environment in step 3.
\end{abstract}

Keywords: housing \& daily living, service robotics, ADLs, vision systems, robotic room

\section{INTRODUCTION}

Experts and masterminds as for example Bill Gates announce the era of service robotics and estimate that service robotics as part of assisted environments will, also because of demographic changes, undergo a similar fast and rigid development as the spread of personal computers in private and economic areas since the 1990s. Already in 1961 Joe Engelberger wondered if relegating robotic technologies explicitly to industrial applications make sense. "The biggest market will be service robots" ${ }^{4}$ asserted Engelberger, who started the industrial robotics era when his firm (Unimation) delivered GM's first robot. Today, robots and distributed robotic subsystems start to permeate our every day surrounding, homes and city environment enhancing them with services and additional performance. At the same time, this permeation is on the way to transform our perception of what robots are, robot technology, robot possibilities and the environment they are merged with. This transformation which has to be understood as a natural part of the evolution of robotics will especially become visible when robots enter the field of service and assistance. Therefore, this article examines new fields of applications for robotics in assistive environments and accompanying transformations. In order to enhance the acceptance of service robotics the proposed approach follows a heavy distribution of sensor-actuator systems into environments and integrates robotic service systems in a seamless or even invisible manner.

\section{Literature Review}

Since the 1980s several research groups have created environments and prototype buildings for socalled smart buildings. Based on Ken Sakamuras TEngine Hardware and a complementary operating system, the Tron House 1, 2 and 3 have been built ${ }^{1}$. The US AwareHome ${ }^{5}$ and PlaceLab ${ }^{6}$ follow a similar approach and MIT's House_ $n^{7}$ includes even modular intelligent furniture that can be equipped with various sensor systems. Recently designed German prototypes of assistive homes, such as "Haus der Gegenwart" (house of presence) ${ }^{8}$ and "Haus der Zukunft" (house of the future) ${ }^{9}$, are exemplarily equipped with a variety of networked pervasive technologies integrated by modern design. Similar to our approach Smart Buildings and Robotic Rooms 
try to integrate sensor-actuator systems with architectonic elements. Smart Buildings can make these systems quite invisible but their performance is limited to more "passive" services that support cognition, energy control, logistics, safety and security.

\section{Research Question and Method}

Buildings and environments consist of a multitude of subsystems as for example walls, ceilings, furniture, service cores and many others. In order to realize the proposed vision of seamless robotic assistance,

Table 1. Overview of proposed experimental approaches according to categories

\begin{tabular}{|c|c|c|c|c|c|c|c|c|c|}
\hline $\begin{array}{c}\text { Sub- } \\
\text { system }\end{array}$ & Wall & Ceiling & $\begin{array}{c}\text { Service } \\
\text { Core }\end{array}$ & Cabinet & Seat & $\begin{array}{c}\text { In- } \\
\text { House } \\
\text { Mobility }\end{array}$ & $\begin{array}{c}\text { Bath- } \\
\text { room }\end{array}$ & $\begin{array}{c}\text { Combined } \\
\text { In-/Outdoor } \\
\text { Mobility }\end{array}$ & $\begin{array}{c}\text { Integrated } \\
\text { Robotic } \\
\text { Environ- } \\
\text { ment }\end{array}$ \\
\hline Project & $\begin{array}{c}\text { Robotic } \\
\text { Service } \\
\text { Wall- } \\
\text { Research } \\
\text { Project } \\
\text { LISA }\end{array}$ & $\begin{array}{c}\text { Modular } \\
\text { Ceiling } \\
\text { Robot }\end{array}$ & $\begin{array}{c}\text { Robotic } \\
\text { Service } \\
\text { Core }\end{array}$ & $\begin{array}{c}\text { Robotic } \\
\text { Cabi- } \\
\text { nets }\end{array}$ & $\begin{array}{c}\text { Robotic } \\
\text { Chair - } \\
\text { Research } \\
\text { Project } \\
\text { GEWOS }\end{array}$ & $\begin{array}{c}\text { Robotic } \\
\text { In- } \\
\text { House } \\
\text { Trans- } \\
\text { fer }\end{array}$ & $\begin{array}{c}\text { Auto- } \\
\text { mated } \\
\text { Bath- } \\
\text { room }\end{array}$ & $\begin{array}{c}\text { Research } \\
\text { Project: } \\
\text { PASSAge }\end{array}$ & AIR LAB \\
\hline Phase & \multicolumn{70}{c|}{ Step 1: 2008-2011 } & Step 2: 2011-2012 & $\begin{array}{c}\text { Step 3: } \\
2012\end{array}$ \\
\hline
\end{tabular}

Activities of Daily Living (ADLs) ${ }^{10}$, which represent tasks in everyday living people need to be able to manage as independent adults, are in most cases not considered or supported by smart buildings realizations. Robotic Rooms ${ }^{11}$ on the other hand can cover the support of more physical activities through robotic systems. However, service robots and mobile robotic platforms are visible, and some sub-systems can be recognized by the supported person as robots. Approaches in the area of Ambient Assisted Living (AAL) mainly focus on health monitoring and patient-physician related communication. Thus, the integration of Digital Communication Frameworks within the home environment comprises an active subject of many ongoing research projects, whereas the integration of Robotic Systems into the home environment is completely neglected.

Furthermore, AAL is mainly driven by the Informatics and Electronics industries without a focus on real architectonic integration. Most projects in that area work with add-on solutions as for example wearable sensors and set-top boxes that have no relation to room configurations or functional layouts. The proposed approach combines elements of Smart Buildings, Robotic Rooms and AAL technologies. However, unlike Robotic Room approaches, this one deals with fully integrating distributed robotic systems with subsystems of buildings, enabling the passive services as well as the hard physical services through mechatronic systems and robotics, to become invisible. In most cases this requires an understanding of living processes, the design of the environment and the design of embedded systems. since 2008, subsystems were investigated in order to obtain insights on how they could be efficiently integrated with mechatronic and robotic elements. This was performed by an interdisciplinary team of researches with backgrounds in architecture, design, electrical engineering, mechanical engineering, informatics and health related sciences. Currently the research team enters Step 3. In this paper building subsystems that have been integrated with mechatronic and robotic elements during step 1 are presented. In the following sections the basic concepts are described in order to explain which show potential for delivering extended services, especially for the ageing society, through this integration. Additionally the core technologies are outlined as well as the design concepts which were utilized to facilitate integration. Finally, it is shown that the ultimate goal is to connect these robotic subsystems to a totally integrated robotic environment (Table 1).

\section{EXPERIMENTAL APPROACHES ACCORDINg to CATE- GORY}

In this section the proposed experimental approaches are presented and the potentials for delivering extended services are outlined. The conducted experiments are categorized into Table 2. Eight different applications are addressed, aiming in providing assistance in the home environment, especially focused in elderly people situations.

Frail older people face numerous challenges in performing ADLs. Simple tasks for a healthy individual are projected under a different perspective to elderly people. Usually, according to the physical condition of the individual, they require the assistance of an extra person in order to perform basic tasks, such as 
bathing, eating, dressing undressing etc. This decreases the independence of elderly people. The proposed research focuses on service delivery, by the integration of robotic elements, mechatronic devices and technologies, which would increase the independence of elderly people in performing basic ADLs. Such assistive technologies could thus greatly enhance the quality of living.

Table 2. Overview own experimental approaches according to categories

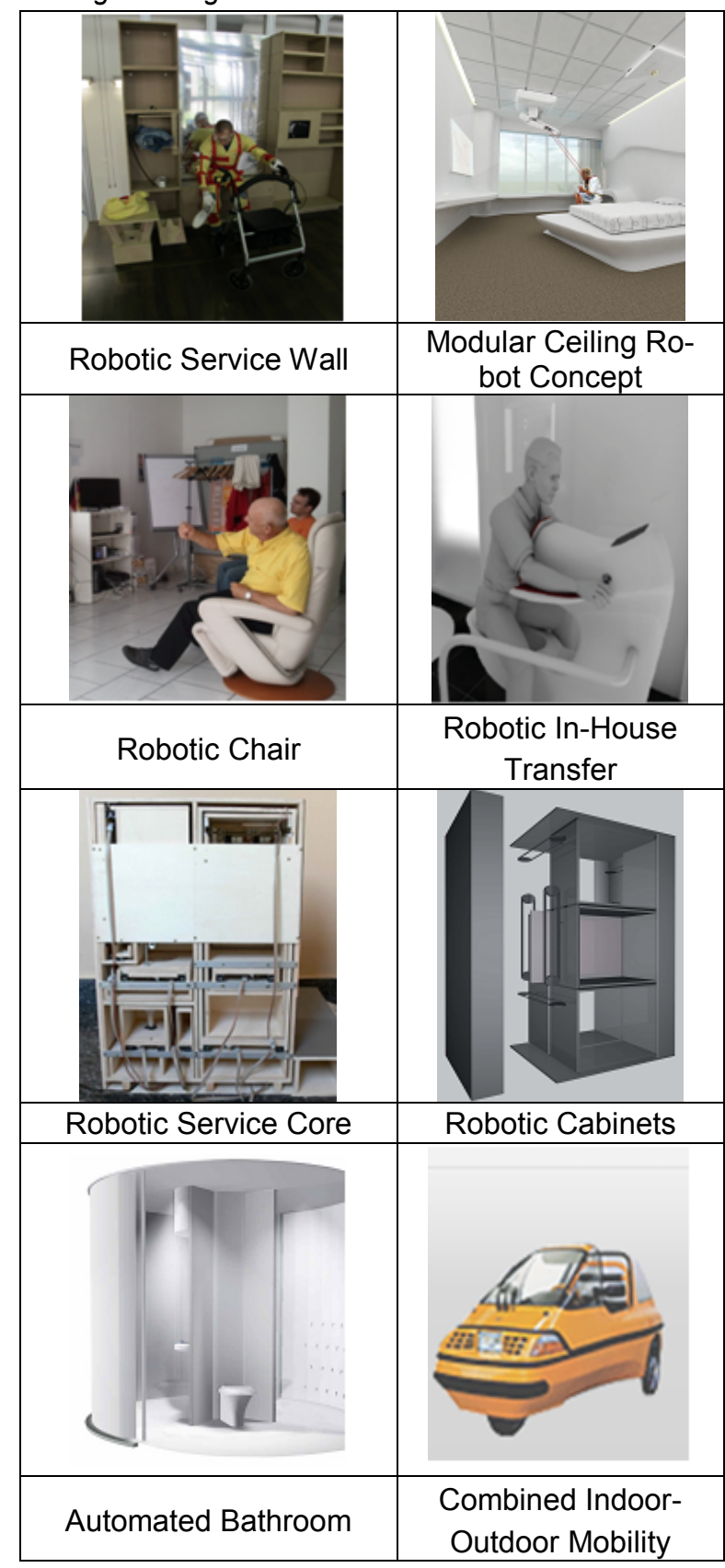

\section{Robotic Service Wall}

The Robotic Service Wall is implemented under a two year funded research project called LISA "Living Independently In South Tyrol Alto Adige" (July 2010July 2012). The consortium consists mainly of industry partners with Technical University of Munich as center point of the consortium both in terms of strat- egy and technology development. In LISA, the implementation of a novel Robotic Service Wall supporting ADLs is the main objective. The proposed system followed a modular approach, whereas all system elements provide "plug and play" characteristics. Robotic actuators, sensors and display screens are populating the proposed robotic wall, in order to enable an Ambient Assisted Living approach. The user can voice command the robotic wall in order to initiate a service or to acquire information on the provided services. The proposed system can be arranged and re-arranged into various configurations, and can be easily installed in any residence without requiring specific space dimensions.

\section{Modular Ceiling Robot Concept}

The purpose was to develop, by a combination of architectural and robotic elements, a system that assists elderly people to live self-sufficient at home. Unlike many other assistance systems our system exploits the ceiling space in order to avoid collisions with conventional living functions. A ceiling suspended robotic system is proposed, navigating with the aid of a grid type rail system among various rooms within a residence. This enables efficient space utilization since it occupies the redundant area of the ceiling. Various types of services can be provided by the proposed system such as health monitoring, infotainment, communications, problem solving, movement assistance, etc.

\section{Robotic Chair}

The implementation of the robotic chair is supported by a funded R\&D-project in the field Ambient Assisted Living called GEWOS. The main objective was to develop a chair that is equipped with invisible but sophisticated sensor technology that measures a multitude of user's vital signs. This inconspicuously and at first glance invisibly integrated sensor modules form the basis for professional activity and sports concepts, health assistance, business and service concepts that are delivered as "services" through the chair and its interface as channel to the users, which through the sensor systems actually becomes part of the system. Therefore GEWOS considers the sensor chair as a socio-technical system, comprising an internet-based platform, an interface component, and further interaction elements and matching services. By encouraging movement and supporting further constitutional methods, this system serves as a health promoter, embedded in the physical and emotional surrounding of the user.

\section{Robotic In-House Transfer}

The proposed approach dealt with identifying the needs and requirements elderly people face in performing ADLs, by conducting a survey among various age groups and identifying the main bottlenecks 
and limitations. A design procedure was then proposed in order to provide an efficient solution in optimizing the independence in functional transfers (getting into and out of bed or wheelchair, getting onto or off toilet, etc.) of elderly people within the home environment. The proposed concept comprises the combination of robotic and architectural elements, aiming at the realization of a novel indoor mobility device enabling independent living, efficiently addressing the mobility difficulties elderly people face within the home environment.

\section{Robotic Service Core}

The prefabricated Service Core is a unit which could be integrated into apartments as a "room within a room" system (Fig. 06???). With its standardized height of $235 \mathrm{~cm}$, it could be positioned in an apartment in an old building as well as in a newly built house. Similar to prefabricated bath or toilet-units for high rise buildings, which have been used in building industry since the 1970s, the service core could be delivered to the site as a prefabricated kit. The Service Core is designed for people with severe disabilities and cognitive diseases. The service core offers most functionalities and appliances which are needed to serve severely handicapped persons who remain indoors. Minimum movement is required to perform daily tasks, since bath and kitchen can be directly and ergonomically reached from the bed area, which can be transformed into a seat. Within the service unit, the health condition of the handicapped person is continuously measured and digital cameras allow relatives, physicians or service personnel to observe the patient and check the current condition in real-time or in regular time intervals. For health monitoring various sensor-based devices are installed in the service core. Additionally the ability of relatives or a physician to communicate with the individual is provided via an integrated communication screen interfaced via internet. The service room can be considered as a very compact hospital or care facility deployed at home. Its aim is to provide severe disabled individuals the possibility to remain as long as possible at their home environment, through the fusion of functional architecture, embedded microcontrollers and ambient intelligence technologies, within an enhanced performance compact and modular service unit. An implemented smaller scale prototype ${ }^{12}$ was realized and implemented for evaluating and testing the overall concept (Table 2).

\section{Robotic Cabinets}

Ergonomics is the engineering science that is concerned with the physical and psychological relationship between machines and the people who use them. Furniture is designed to adapt to specific needs, supporting various human activities. According to the application, a set of guidelines or rules is followed during design, in order to provide ergonomic features. The proposed robotic cabinet system provides an integrated solution, comprising reduced space utilization, modularity, and intelligent operation while remaining an ergonomic piece of furniture. . A set of electrical motors is used to position the various sections of the system into the correct height level, and to displace them towards the horizontal axis, to allow a rotational motion path. The implemented prototype was evaluated in terms of, ergonomics (using an age simulation suite), organizational ergonomics within a room, and space utilization (as the individual sections/shelves can rotate in a vertical direction, the proposed system is possible to be installed in small rooms, achieving a $20 \%$ less space utilization usage, in order to allow free space for mobility with rollators or wheelchairs).

\section{Automated Bathroom}

An approach towards robotic hygienic assisted services focusing in the global demographic change problem is addressed by the proposed research. The proposed approach dealt with identifying the needs and requirements by conducting a survey among various age groups and identifying the main bottlenecks and limitations. A design procedure was then proposed in order to provide an efficient solution in optimizing hygienic services in the home environment while increasing the independence of elderly people in the corresponding ADLs. The proposed concept comprises the combination of robotic and architectural elements, aiming at the realization of a novel robotic bathroom which can automate the bathing procedure.

\section{Combined Indoor-Outdoor Mobility}

The implementation is supported by a funded R\&Dproject in the field Ambient Assisted Living called PASSAge. The PASSAge addresses the mobility issues within an ageing society by developing a modular and personalized mobility system, that can be integrated into the individual surrounding of the user and encourages individual mobility as well as supports safety, comfort and health, and therefore enhances quality of living. Adaptable, customizable and user-friendly add-on modules (operation and shopping help, health phones, transfer support at the entrance of buildings or within buildings) are being adapted from existing technologies and innovative mobility components in the field of personal mobility devices.

\section{Core TeChNologies \\ Using ROS/GAZEBO as Middleware}

Building Information Modeling is one of the recent developments in architecture, construction and engineering which helps to visualize, support and manage buildings during design construction and opera- 
tion. The developments in the field of pervasive computing though made the buildings more complex making the current Building Information Modeling $(\mathrm{BIM})^{13}$ tools obsolete, as they do not contain functionality to integrate sensor data and other pervasive technologies. Also in a pervasive environment there is a need for the building model to interact with the surroundings in real-time. Mahdavi's research on sentient buildings ${ }^{14}$ illustrates that for a building to be intelligent and proactive, the building model should be able to read inputs from a wide array of sensory networks, and update itself on real-time. Thus, the requirement to access and control the various sensors and computers in a ubiquitous environment introduces the need for a middleware.

Robot Operating System (ROS) ${ }^{15}$ is one such middleware, able to enable control over the various sensors and exchange data information in real-time. ROS can be used along with a 3-D visualization simulator environment called Gazebo ${ }^{16}$ to create a model for a ubiquitous environment. Modeling in Gazebo is done by considering the building as an immobile robot with various joints and links. Creating a model using the concept of joints and links enables the building model to be more dynamic and updated in real time based on the sensor information. With proper mechatronic systems this also gives the option to control the dynamic parts of the building from a remote location. Also this ROS/Gazebo integration is more suited to an environment cohabited with robots as shown in Figure 1 where these tools simplify the complexity required for the robot in order to successfully navigate the architectural space and perform the required tasks.

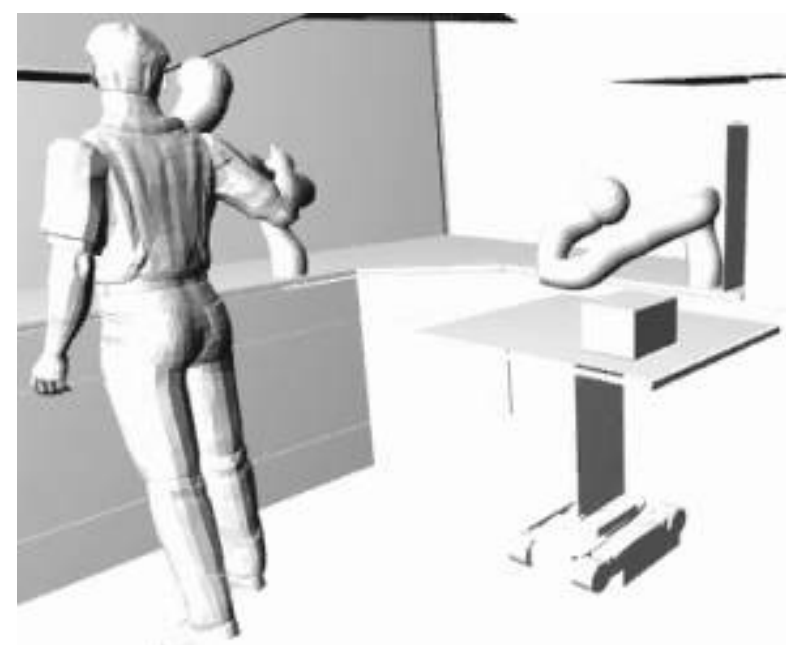

Fig.1. Robotic environment modeled in Gazebo using concept of links and Joints

\section{RFID Technology}

In recent years, radio frequency identification technology (RFID) has moved from insignificance into conventional applications that aid in simplifying the handling of items and objects. RFID enables identifi- cation from a distance, and unlike earlier bar-code technology, it functions without requiring a line of sight or a specific visual pattern to be detected, recorded and processed ${ }^{17}$. RFID tags (see Figure 2) support a larger set of unique IDs than bar codes and can incorporate additional data such as manufacturer, product type, and even measure environmental factors such as temperature. Furthermore, RFID systems can discern many different tags located in the same general area without human assistance. In contrast, consider a supermarket checkout counter, where the personnel must orient each barcoded item towards a laser scanner reader in order to identify it. If all items had an RFID tag attached on them, the checkout process on the counter could have been fully automated without explicitly requiring human assistance.

Many types of RFID exist, but at the highest level, we can divide RFID devices into two broad classes ${ }^{18}$ : active and passive. Active tags require a power source, i.e. they are either connected to a powered infrastructure or use energy stored in an integrated battery. One example of an active tag is the transponder attached to an aircraft that identifies its national origin. However, batteries make the cost, size, and lifetime of active tags impractical for most small scale applications. Passive RFID tags are thus preferred because they don't require an external supply source. The tags also have huge operational lifetime and are tiny enough to fit into a practical adhesive label.

A passive tag consists of three parts: an antenna, a processing unit attached to the antenna, and some form of encapsulation. A tag reader is responsible for powering and communicating with a tag, which is attached either to a personal computer or to a digital communication network. The tag antenna captures energy and transfers the tag's ID. The tag's processing unit is responsible for coordinating the communication and transmission process. The encapsulation maintains the tag integrity and protects the antenna and processing unit from environmental conditions or damage.

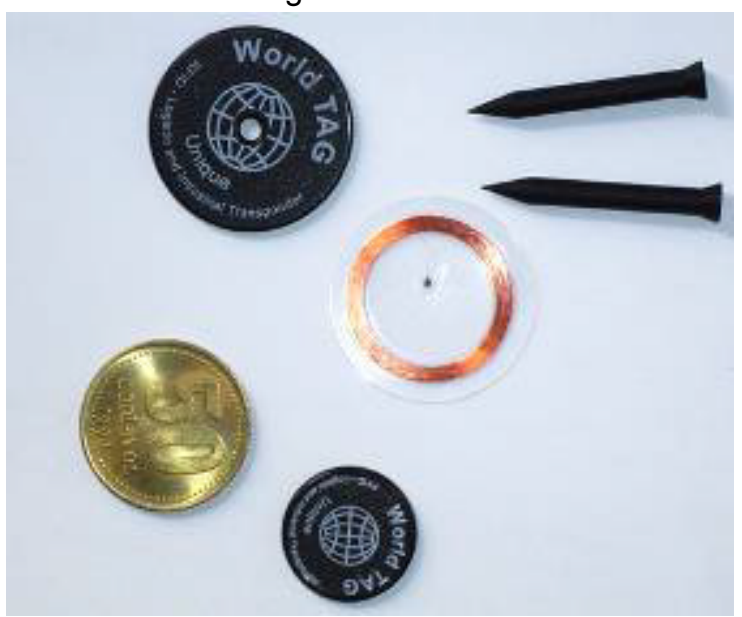

Fig.2. RFID tags 
In a home environment this technology can be utilized to assist elderly people by providing them a real-time inventory of their high priority items. Experiments conducted during the proposed study, revealed the efficiency of this "invisible" technology, and the variety of potential applications that this technology can contribute to. By combining computerized databases and inventory control, linked through digital communication networks spread across the home environment and across a global set of locations, RFID technology can efficiently pinpoint individual items as they move between locations, warehouses, vehicles, and stores.

\section{Vision Systems}

Depth perception is one of the important tasks of a machine vision system. Stereo correspondence by calculating the distance of various points in a scene relative to the position of a camera allows the performance of complex tasks, such as depth measurements and environment reconstruction ${ }^{19}$. The most common approach for extracting depth information from intensity images is by means of a stereo camera setup. The point-by-point matching between the two images from the stereo setup derives the depth images, or the so called disparity maps ${ }^{20}$. Disparity map extraction of an image is a computationally demanding task. Due to the computational complexity involved in stereo vision algorithms, powerful CPUs are required to achieve acceptable performance. Recent research has shown that such low level image processing algorithms can be efficiently implemented in hardware.

The proposed research deals with implementing depth extraction algorithms using stereo camera arrangements or depth sensors, like the "Microsoft Kinect" Sensor ${ }^{21}$. The acquired depth representation of an individual on a chair within the laboratory environment using Microsoft's Kinect depth sensor is depicted in Figure 3. The depth map was acquired using a Turtlebot $^{22}$ mobile rover, which an onboard Microsoft depth sensor. The mobile rover can be seen in Figure 4.

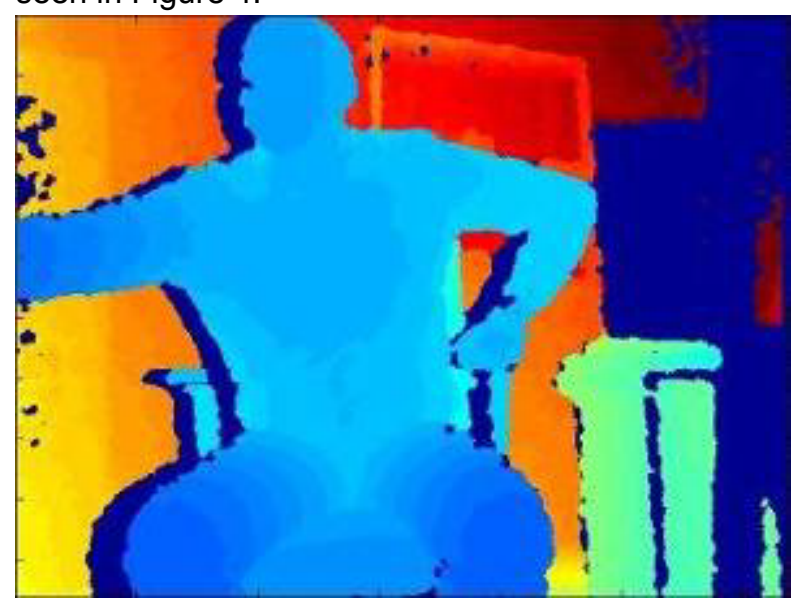

Fig.3. Acquired depth representation
Depth perception allows the autonomous navigation of mobile robots, object recognition and people detection. These implementations aim to integrate an efficient vision system in the home environment, in order to allow systems to be operated by people gestures or movements. Additionally the presence of a person within different areas of an apartment can accordingly trigger the deployment of robotic elements and mechatronic devices, in order to allow an Ambient Intelligence environment realization.

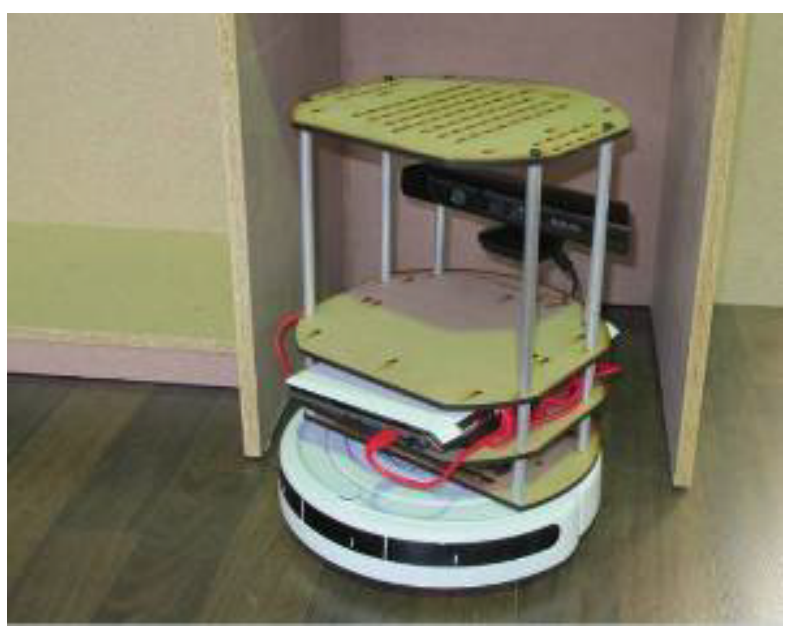

Fig.4. TurtleBot mobile rover

\section{CONCLUSIONS AND FUTURE WORK}

New application fields for robotics in the area of service and environmental assistance are generated through the tension between market pull on the one hand and technology push on the other. However, the integration of robotic systems and sub-systems is likely to transform the appearance of our environment and everyday artifacts similar as done through the implementation of new materials or technologies before.

The integration and permeation of our environments and social spaces with robotic technology and related sub-systems will lead to new concepts and explore new forms as robotic walls, robotic furniture, robotic care environments, mobility robots and robotic everyday companions. Obviously emerging technologies are not only transforming functionality of upcoming hybrid robot-environment systems but also designs and forms of our environment following the principle of "form follows function" (Sullivan, 1896) being discovered and scientifically explained by L. Sullivan more than one hundred years ago.

Despite all complex interdependencies and transformations, underlying principles as modularity, customization capability, system distribution, standardization and a well defined human-robot relation seem to be helpful tools in designing future hybrid robotenvironment systems. Above that, modularity and standardization are important prerequisites for industrially fabricated and customized robotic enhanced 
assistive environments. Complex and multilevel hightech environments will only reach affordability and long-term serviceability when fabricated with frontier industrial technology.

Furthermore, tools like ROS and Gazebo have become more important in light of new building concepts with embedded robotic systems. Over centuries buildings have always been viewed as static structures resistant to changes. However, more recently there have been a different viewpoint about buildings, and they are often viewed as kinetic structures capable of changing its structure or profile to suit the various environmental or user conditions. The concept of kinetic architecture or dynamic buildings is currently being exploited by many architects around the globe. Some projects use these concepts to make the building more sustainable and intelligent while other projects employ these concepts for assistance. We use embedded robotics to build up robotic service environments that are completely integrated in our environment to support activities of daily living. Currently a modular, 1:1 scale robotic testing flat is finalized for experimenting at the author's robotic laboratory, Figure 5 . In this environment the aforementioned systems shall be integrated to a total robotic environment.

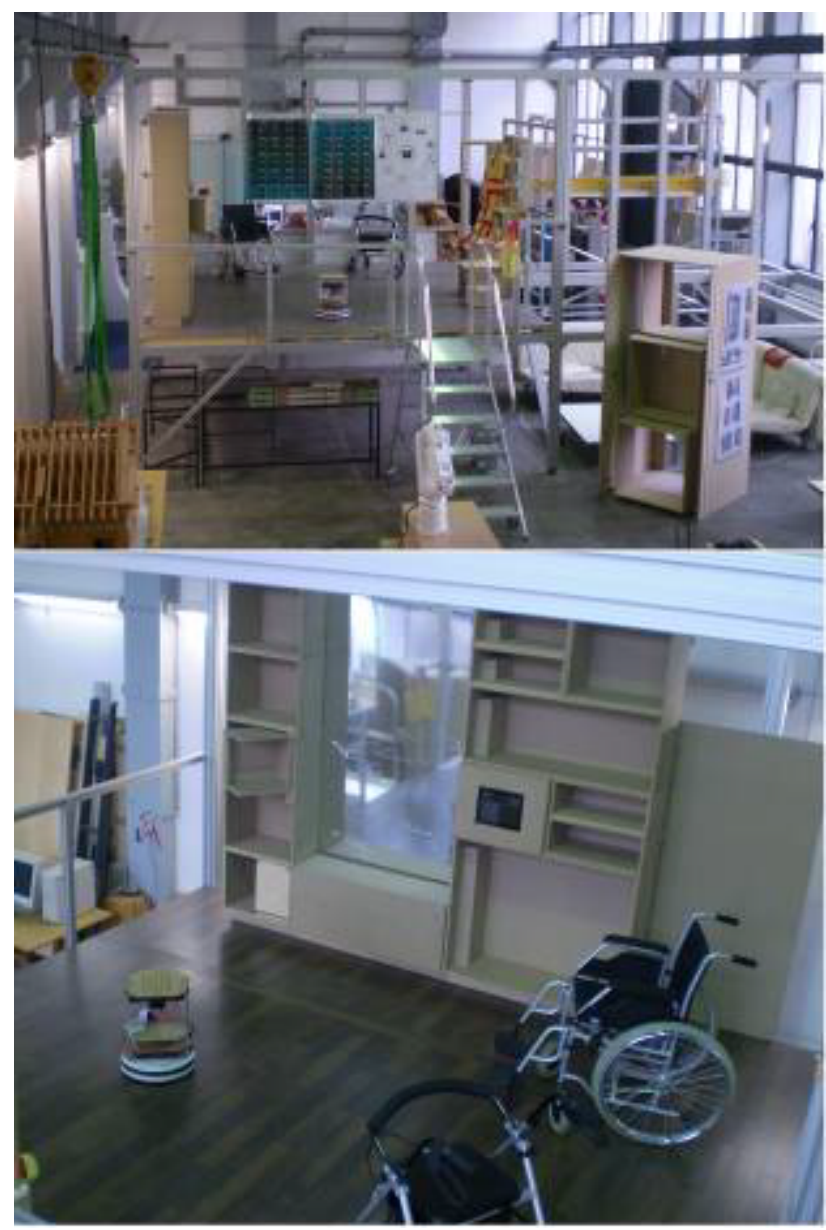

Fig.5. 1:1 scale robotic testing flat
The proposed research is not focusing on integrating mainly sensors, actuators and robots on an informational level, nor oriented to implementations that are realized in a controlled experimental environment. The proposed research targets robotic service delivery straightforwardly applied into regular home environments. In order to integrate those services on basis of technologies seamlessly and connect them to $A D L s^{10}$, a fusion scheme of robotic technology physically and informational with architectural elements as e.g. walls and barrier-free design is proposed. The advantage of such an approach is that that existing walls do not have to be replaced.

Analysis showed that a smart wall which would replace existing walls would become too complex and too expensive to install. In order to keep cost and effort low enough for equipping a flat with smart technology, it was proposed to concentrate assistive technology on terminals instead of developing a smart wall. Each terminal can be deployed in front of an existing wall as a pre-fabricated kit. A terminal concentrates assistive functions and technologies that have to be brought into a room on a compact element. Thus, in case a room has to be equipped with assistive technology, not the whole room has to be rearranged or renovated, but only one of the proposed compact elements has to be installed in this room as an add-on.

\section{References}

1. Shimizu, N., "A House of Sustainability: PAPI: Intelligent House in the Age of Ubiquitous Computing", Architecture and Urbanism (AU), Special Issue, 2005.

2. Krumm, J., Ubiquitous Computing Fundamentals, CRC Press, 2010.

3. Siciliano, B., Sciavicco, L., Villani, L., Oriolo, G., Robotics - Modelling, Planning and Control, Springer, 2010.

4. Englberger, J.F., Robotics in Service, Massachussets, MIT Press, 1989.

5. Kidd, C.D., Orr, R., Abowd, G.D., Atkeson, C.G., Essa, I.A., Maclntyre, B., Mynatt, E.D., Starner, T., Newstetter, W., "The Aware Home: A Living Laboratory for Ubiquitous Computing Research", Proceedings of the Second International Workshop on Cooperative Buildings, Integrating Information, Organization, and Architecture, pp. 191-198, 1999.

6. Initlle, S., Larson, K., Tapia, E.M., Beaudin, J., Kaushik, P., Nawyn, J., Rockinson, R., “Using a Live-in Laboratory for Ubiquitous Computing Research", Intl. Conf. on Pervasive Computing, pp. 349-365, 2006.

7. Larson, K., Stephen, I., MIT Open Source Building Alliance - $A$ house_ $n$ initiative, Position Paper, MIT House_n, 2005.

8. Haus der Gegenwart, Munich, Germany. Partners: Microsoft, BMW, Munich City. Website, last visited 
12.11.2009,

http://www.haus-der-

gegenwart.de/partner/

9. Intelligent Networking: T-Com House. Siemens, Telecom Laboratories. Berlin, 2005-2006.

10. Activities of Daily Living Evaluation, Encyclopedia of Nursing \& Allied Health. ed. Kristine Krapp. Gale Group, Inc., 2002.

11. Sato, T., Harada, T., Mori, T., "Environment-type robot system "RoboticRoom" featured by behavior media, behavior contents, and behavior adaptation", IEEE Transactions on Mechatronics, Vol. 9(3), pp. 529-534, 2004.

12. Linner, T., Kranz, M., Roalter, L., Bock, T., "Compacted and industrially customizable ambient intelligent service units: Typology, examples and performance", in 2010 Sixth International Conference on Intelligent Environments, Kuala Lumpur, Malaysia, pp. 295-300, 2010.

13. Eastman, C., Teicholz, P., Sacks, R., Liston, L., BIM Handbook - A guide to building information modeling for owners, managers, designers, engineers and contractors, 2008.

14. Mahdavi, A., "The technology of sentient buildings", ITU A|Z, Vol. 3(172), pp. 25-36, 2006.

15. ROS, http://www.ros.org/wiki/ROS

16. Gazebo, http://gazebosim.org/about.html

17. Finkelzeller, K., The RFID Handbook, $2^{\text {nd }}$ edition, John Wiley \& Sons, 2003.

18. Want, R., "An introduction to RFID technology", IEEE Pervasive Computing, Vol. 5(1), pp. 25-33, 2006.

19. Jain, R., Kasturi, R., Schunck, B.G., Machine Vision, $1^{\text {st }}$ edition, McGraw-Hill, New York, 1995.

20. Faugeras, O., Three Dimensional Computer Vision: a geometric viewpoint, MIT Press, Cambridge, MA, 1993.

21. Microsoft Kinect, http://en.wikipedia.org/wiki/Kinect

22. Turtlebot, http://turtlebot.com 